\title{
Imagery and retrieval of auditory and visual information: Neural correlates of successful and unsuccessful performance
}

\author{
Willem Huijbers a,b,*, Cyriel M.A. Pennartz ${ }^{\mathrm{a}}$, David C. Rubin ${ }^{\mathrm{c}, \mathrm{d}}$, Sander M. Daselaar ${ }^{\mathrm{a}, \mathrm{c}}$ \\ a University of Amsterdam, Swammerdam Institute for Life Sciences, Faculty of Science, Amsterdam, The Netherlands \\ ${ }^{\mathrm{b}}$ Martinos Center for Biomedical Imaging, Massachusetts General Hospital, Department of Neurology, Brigham and Women's Hospital, Boston, MA, USA \\ ${ }^{\mathrm{c}}$ Duke University, Department of Psychology and Neuroscience, Duke University, Durham, NC, USA \\ ' Center on Autobiographical Memory Research, Aarhus University, Aarhus, Denmark
}

\section{A R T I C L E I N F O}

\section{Article history:}

Received 3 June 2010

Received in revised form 21 February 2011

Accepted 26 February 2011

Available online 9 March 2011

\section{Keywords:}

Episodic memory

Imagery

fMRI

Visual

Auditory

\begin{abstract}
A B S T R A C T
Remembering past events - or episodic retrieval - consists of several components. There is evidence that mental imagery plays an important role in retrieval and that the brain regions supporting imagery overlap with those supporting retrieval. An open issue is to what extent these regions support successful vs. unsuccessful imagery and retrieval processes. Previous studies that examined regional overlap between imagery and retrieval used uncontrolled memory conditions, such as autobiographical memory tasks, that cannot distinguish between successful and unsuccessful retrieval. A second issue is that fMRI studies that compared imagery and retrieval have used modality-aspecific cues that are likely to activate auditory and visual processing regions simultaneously. Thus, it is not clear to what extent identified brain regions support modality-specific or modality-independent imagery and retrieval processes. In the current fMRI study, we addressed this issue by comparing imagery to retrieval under controlled memory conditions in both auditory and visual modalities. We also obtained subjective measures of imagery quality allowing us to dissociate regions contributing to successful vs. unsuccessful imagery. Results indicated that auditory and visual regions contribute both to imagery and retrieval in a modality-specific fashion. In addition, we identified four sets of brain regions with distinct patterns of activity that contributed to imagery and retrieval in a modality-independent fashion. The first set of regions, including hippocampus, posterior cingulate cortex, medial prefrontal cortex and angular gyrus, showed a pattern common to imagery/retrieval and consistent with successful performance regardless of task. The second set of regions, including dorsal precuneus, anterior cingulate and dorsolateral prefrontal cortex, also showed a pattern common to imagery and retrieval, but consistent with unsuccessful performance during both tasks. Third, left ventrolateral prefrontal cortex showed an interaction between task and performance and was associated with successful imagery but unsuccessful retrieval. Finally, the fourth set of regions, including ventral precuneus, midcingulate cortex and supramarginal gyrus, showed the opposite interaction, supporting unsuccessful imagery, but successful retrieval performance. Results are discussed in relation to reconstructive, attentional, semantic memory, and working memory processes. This is the first study to separate the neural correlates of successful and unsuccessful performance for both imagery and retrieval and for both auditory and visual modalities.
\end{abstract}

(c) 2011 Elsevier Ltd. All rights reserved.

\section{Introduction}

Mental imagery - perceiving with the mind's eye and ear is considered to be a critical component of vivid remembering of past events - or episodic retrieval (Baddeley, 1992; Greenberg \& Rubin, 2003). Theories of memory hold that episodic retrieval does not only involve the activation of previously formed memory traces, but also reconstructive processes that support the gener-

\footnotetext{
* Corresponding author. Tel.: +1 6177265573.

E-mail address: huijbers@nmr.mgh.harvard.edu (W. Huijbers).
}

ation of mental representations (Bartlett, 1932; Daselaar et al., 2008; Rubin, Schrauf, \& Greenberg, 2003; Tulving, 1983). In line with these theories, recent functional MRI (fMRI) studies have indicated that imagery and retrieval show overlapping activations in several regions including the hippocampus, medial prefrontal cortex (mPFC), posterior midline region (PMR) and the angular gyrus of the ventral parietal cortex (Addis, Pan, Vu, Laiser, \& Schacter, 2009; Addis, Wong, \& Schacter, 2007; Buckner \& Carroll, 2007; Hassabis \& Maguire, 2007). However, these studies used uncontrolled, open-ended autobiographical memory tasks to examine the neural correlates of memory, which have no objective measures of successful vs. unsuccessful performance (Cabeza \& St Jacques, 
Table 1

Task setup and data used in the fMRI analyses.

\begin{tabular}{|c|c|c|c|c|c|c|}
\hline \multirow[t]{2}{*}{ Condition } & \multicolumn{3}{|l|}{ Day 1} & \multicolumn{3}{|l|}{ Day 2} \\
\hline & Timing & Imagery task & Data used & Timing & Retrieval task & Data used \\
\hline \multirow[t]{3}{*}{ Imagery } & $1.0 \mathrm{~s}$ & Cue word & \multirow[t]{3}{*}{ Included } & $4.0 \mathrm{~s}$ & Retrieve imagined & \multirow[t]{3}{*}{ Not included } \\
\hline & $3.0 \mathrm{~s}$ & Imagine image or sound & & & Event from day $1^{\mathrm{b}}$ & \\
\hline & $1.5 \mathrm{~s}$ & Rate richness (1-4) & & $1.5 \mathrm{~s}$ & Rate confidence (1-2) & \\
\hline \multirow[t]{3}{*}{ Perception } & $1.0 \mathrm{~s}$ & Cue word & \multirow[t]{3}{*}{ Not included $^{\mathrm{a}}$} & $4.0 \mathrm{~s}$ & Retrieve perceived & \multirow[t]{3}{*}{ Included } \\
\hline & $3.0 \mathrm{~s}$ & Perceive image or sound & & & Event from day $1^{\mathrm{b}}$ & \\
\hline & $1.5 \mathrm{~s}$ & Rate quality (1-4) & & $1.5 \mathrm{~s}$ & Rate confidence $(1-2)$ & \\
\hline
\end{tabular}

a Perceptual trials are used for the modality-specific analysis only.

b Note that both retrieval and confidence rating required a response.

2007; Cabeza et al., 2004; Cabeza \& Nyberg, 2000). Moreover, the subjective quality of the imagery experience (successful vs. unsuccessful imagery) was generally not assessed (see however Addis et al., 2007). Thus, the overlap between brain regions that support successful vs. unsuccessful imagery and retrieval remains unclear.

A second issue is that fMRI studies that compared imagery and retrieval have used general cues that did not emphasize a specific modality (e.g. Addis et al., 2009; Addis et al., 2007; Hassabis, Kumaran, \& Maguire, 2007; Szpunar, Watson, \& McDermott, 2007). Such cues are likely to activate both auditory and visual processing regions simultaneously. Thus, without word cues that are specific to the auditory or visual modality, it remains unclear to what extent previously identified brain regions truly support modalityindependent imagery and retrieval processes.

To address these issues we conducted an fMRI experiment that consisted of a perceptual task, an imagery task with subjective performance ratings and a controlled retrieval task with objective memory measures. Moreover, to assess the modalityindependence of regions, each task included both auditory and visual conditions. As shown in Table 1, on Day 1, participants imagined sounds and pictures based on imagery word cues. In addition, they also perceived sounds and pictures following different word cues. Imagery-related activity was assessed by using imagery quality ratings following each trial. Successful imagery was defined as more activity for high- than for low-rated imagery experiences and the opposite pattern as unsuccessful imagery. On Day 2, participants retrieved the previously perceived and imagined sounds and pictures based on retrieval word cues. To keep our findings consistent with controlled studies of retrieval
(Buckner \& Wheeler, 2001; Nyberg, Habib, Mcintosh, \& Tulving, 2000), we only assessed the retrieval of externally presented auditory and visual events (Table 1). Retrieval-related activity was assessed by contrasting items that were remembered with high confidence (successful retrieval) to those that were forgotten (unsuccessful retrieval). Regional overlap between imagery and retrieval was assessed by employing a factorial design in which the factors task (imagery/retrieval) and performance (successful/unsuccessful) were crossed for both auditory and visual modalities. Although many fMRI studies have compared activations during successful and unsuccessful retrieval conditions (Buckner \& Wheeler, 2001), to our knowledge this is the first retrieval study to compare these activations with successful and unsuccessful imagery.

In the present study, we tested two main predictions. First, we predicted that in line with previous fMRI studies, imagery and retrieval of auditory and visual information should activate auditory and visual regions respectively (Buckner, Logan, Donaldson, \& Wheeler, 2000; Deacon, Kosslyn, \& Scarry, 2001; Kraemer, Macrae, Green, \& Kelley, 2005). Second, we predicted that, in line with studies that used uncontrolled memory tasks (Hassabis et al., 2007a; Schacter, Addis, \& Buckner, 2007; Szpunar et al., 2007), the hippocampus, posterior cingulate cortex, medial prefrontal cortex and angular gyrus will show a pattern associated both with imagery success and retrieval success. We also expected this pattern to occur regardless of whether the task had an auditory or visual orientation. Although we did not have clear predictions regarding brain regions, we also assessed the other cells of the task $\times$ performance factorial design, shared by both modalities: the opposite main effect (unsuccessful > successful), and

Table 2

overview of behavioral results.

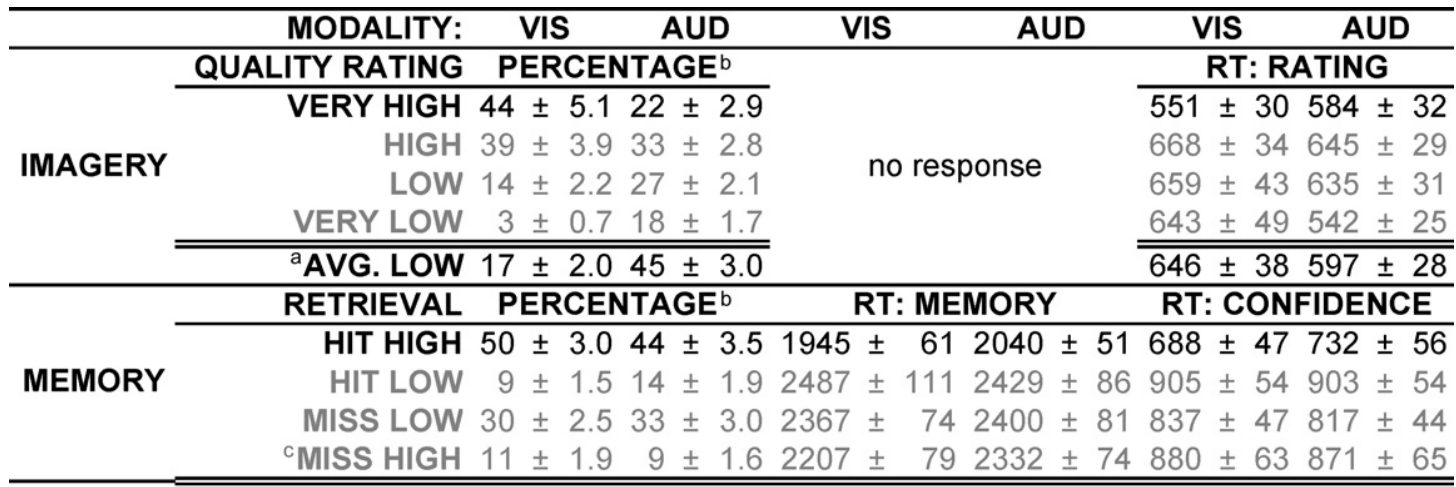

${ }^{a}$ AVG. MISS $41 \pm 2.042 \pm 3.02317 \pm 722380 \pm 72821 \pm 45818 \pm 44$

a Only black values are used in fMRI analysis, AVG: both imagery low/both retrieval miss trials combined.

${ }^{b}$ Note that given the 4 options during retrieval, chance-level is $25 \%$.

${ }^{c}$ Note that MISS HIGH includes missing values. 


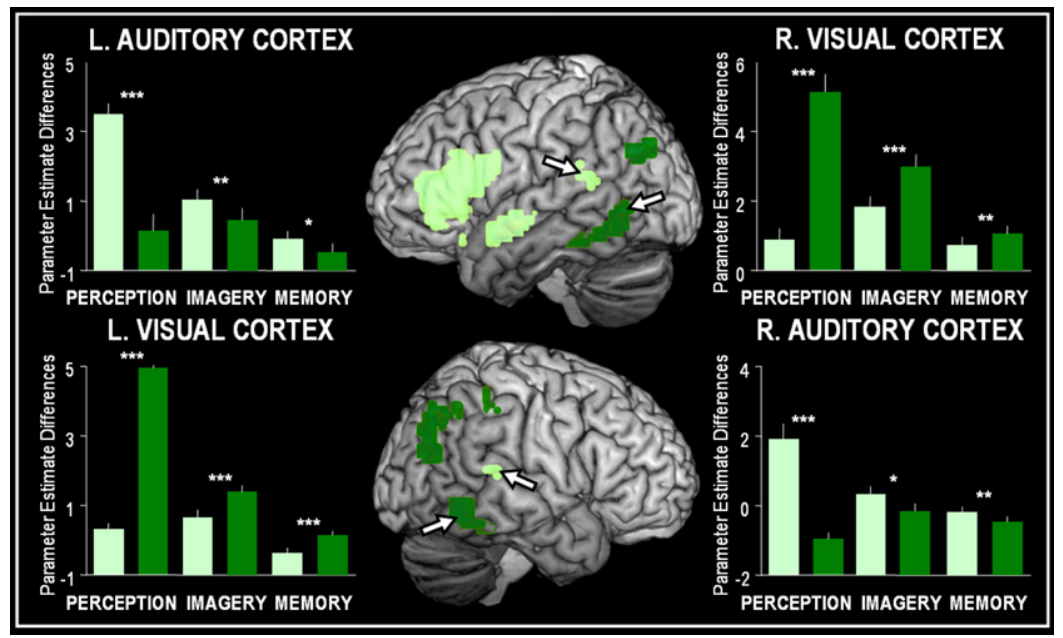

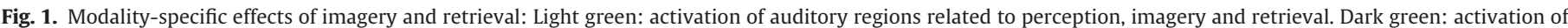

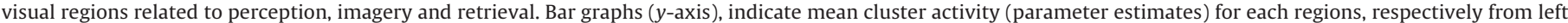

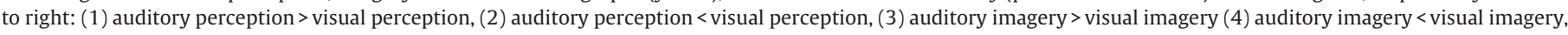

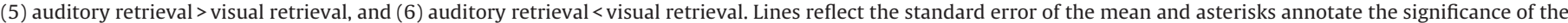
difference between the auditory and visual contrast. Number of asterisks denotes $P$-values $\left({ }^{*} P<0.05,{ }^{* *} P<0.01,{ }^{* * *} P<0.001\right)$.

interactions between task (imagery $\times$ retrieval) and performance (successful $\times$ unsuccessful).

\section{Results}

\subsection{Behavioral data}

Table 2 gives a complete overview of reaction times (RTs) and proportions of responses for each condition. Below, we only report the outcome of the repeated measures ANOVAs concerning the RT and accuracy differences between the trials that were included in the successful (imagery quality rating of 4 ) and unsuccessful (imagery quality ratings of 1 or 2 ) imagery, and successful (high confidence correct) and unsuccessful (incorrect) retrieval contrasts.

Regarding imagery performance, a repeated measures imagery (unsuccessful/successful) $\times$ modality (auditory/visual) ANOVA showed a main effect of performance on RTs $(P=0.0088)$ indicating that the ratings for successful imagery trials were faster than those for unsuccessful imagery trials. Although we found no effect of modality $(P=0.62)$, there was a significant modality $\times$ imagery interaction $(P=0.0098)$ indicating a greater performance difference in RTs for the visual condition. Consistent with the RT results, the percentage of successful items was also significantly lower $(P<0.0001)$ for auditory than for visual imagery suggesting that the former condition was more difficult.

Retrieval trials involved two responses, one during the retrieval period and one during the confidence rating period. During the retrieval period, we found a main effect of memory performance on RTs $(P<0.001)$ reflecting faster RTs for successful than unsuccessful retrieval responses. In addition, we found a significant main effect of modality on RTs $(P=0.023)$ indicating that retrieval of auditory information was slower than retrieval of visual information. However in terms of memory accuracy, there was no effect of modality $(P=0.092)$. During the rating period of the retrieval task, we found a main effect of memory $(P<0.001)$, but not modality $(P=0.13)$, on RTs. We also found a significant modality (auditory/visual) $\times$ memory (successful/unsuccessful) interaction $(P=0.025)$ indicating a greater confidence rating RT difference between successful and unsuccessful responses for the visual than the auditory modality. Overall the behavioral results suggest that auditory imagery was more difficult than visual imagery, but, at least based on the memory accuracy, there was no evidence for a modality difference in difficulty during retrieval.

\section{2. $f M R I$ data}

\subsubsection{Modality-specific contributions to imagery and retrieval}

Following fMRI studies that examined imagery and retrieval separately (Buckner et al., 2000; Deacon et al., 2001; Kosslyn, Ganis, \& Thompson, 2001; Kraemer et al., 2005; Wheeler, Petersen, \& Buckner, 2000), we predicted that imagery and retrieval of auditory and visual information should activate auditory and visual sensory regions, respectively. Confirming this prediction for the auditory modality, we found common activity for auditory perception (perception of sounds $>$ images), auditory imagery (successful auditory imagery > successful visual imagery), and auditory memory retrieval (successful auditory retrieval > successful visual retrieval) within auditory processing regions including bilateral superior temporal gyrus/secondary auditory cortex (Fig. 1 and Table 3). Likewise, confirming our prediction for the visual modality, we found that a region comprising bilateral V3/V4 and fusiform gyrus (Fig. 1 and Table 3 ) was not only activated during visual perception (perception of images $>$ sounds), but also during visual imagery (successful visual imagery > successful auditory imagery), and visual memory retrieval (successful visual retrieval > successful auditory retrieval). These findings confirm the modality-specific role of sensory processing regions in imagery and retrieval of auditory and visual information (Buckner et al., 2000; Deacon et al. 2001; Kosslyn et al., 2001; Kraemer et al., 2005; Wheeler et al., 2000a; Wheeler et al., 2000b).

\subsection{Overlap and interactions between imagery and retrieval in modality-independent regions}

Table 4 and Figs. 2-5 summarize the regions that displayed consistent patterns of imagery and retrieval activity for both auditory and visual modalities. Regions that did not show significant or trending activity for one of the modalities were not included in the table. 
Table 3

Regions showing overlapping activations during perception, imagery and retrieval.

\begin{tabular}{|c|c|c|c|c|c|c|c|c|}
\hline Region & Side & $\mathrm{BA}$ & $X$ & $Y$ & $Z$ & T-PER & T-IMG & T-RET \\
\hline \multicolumn{9}{|l|}{ Auditory $>$ visual } \\
\hline \multirow{2}{*}{ Auditory cortex/superior temporal gyrus } & $\mathrm{L}$ & $22 / 41$ & -60 & -42 & 15 & 12.5 & 2.61 & 2.05 \\
\hline & $\mathrm{R}$ & $22 / 41$ & 48 & -39 & 6 & 7.90 & 2.27 & 3.25 \\
\hline Inferior temporal gyrus & $\mathrm{L}$ & 20 & -42 & -6 & -15 & 11.6 & 5.65 & 7.30 \\
\hline Inferior frontal gyrus & $\mathrm{L}$ & 47 & -54 & 9 & 18 & 8.59 & 8.37 & 6.20 \\
\hline \multirow[t]{2}{*}{ caudate } & $\mathrm{L}$ & - & -15 & 6 & 9 & 6.43 & 3.95 & 3.24 \\
\hline & $\mathrm{R}$ & - & 12 & 6 & -6 & 7.12 & 2.19 & 3.05 \\
\hline \multicolumn{9}{|l|}{ Visual > auditory } \\
\hline \multirow[t]{2}{*}{ Fusiform gyrus } & $\mathrm{L}$ & $36 / 37$ & -27 & -57 & -15 & 15.0 & 5.78 & 6.12 \\
\hline & $\mathrm{R}$ & $36 / 37$ & 27 & -45 & -15 & 23.2 & 4.45 & 5.54 \\
\hline \multirow[t]{2}{*}{ Visual cortex (V3/V4) } & $\mathrm{L}$ & 19 & -42 & -6 & -9 & 11.8 & 7.91 & 4.04 \\
\hline & $\mathrm{R}$ & 19 & 42 & -78 & -15 & 10.1 & 7.88 & 3.15 \\
\hline \multirow[t]{3}{*}{ Parieto-occipital cortex } & $\mathrm{L}$ & $19 / 39$ & -27 & -72 & 24 & 8.55 & 9.40 & 2.98 \\
\hline & $\mathrm{R}$ & $19 / 39$ & 45 & -45 & 51 & 3.83 & 6.74 & 3.18 \\
\hline & $\mathrm{R}$ & $19 / 40$ & 36 & -60 & 42 & 3.61 & 4.11 & 2.84 \\
\hline Parahippocampus & $\mathrm{R}$ & 19 & 12 & -48 & 9 & 5.11 & 4.62 & 5.01 \\
\hline \multirow[t]{2}{*}{ Posterior cingulate cortex } & $\mathrm{L}$ & 30 & -18 & -57 & 12 & 3.08 & 2.92 & 3.08 \\
\hline & $\mathrm{R}$ & 31 & 3 & -33 & 36 & 4.29 & 3.22 & 4.90 \\
\hline
\end{tabular}

$\mathrm{T}$-PER = T-values perception, $\mathrm{T}$-IMG = T-values imagery, $\mathrm{T}$-RET $=\mathrm{T}$-values retrieval .

\subsubsection{Main positive effect: successful imagery and successful retrieval}

As shown in Fig. 3 and in line with our prediction, the hippocampus, posterior cingulate cortex (PCC), medial prefrontal cortex (mPFC) and angular gyrus were associated both with imagery success (successful > unsuccessful imagery) and retrieval success (successful $>$ unsuccessful retrieval) in both auditory and visual modalities (Table 4). These findings confirm results from previous studies showing overlapping activity in these regions during imagery and retrieval (Addis et al., 2007; Addis et al., 2009; Buckner \& Carroll, 2007; Hassabis \& Maguire, 2007). At the same time, the current results extend these findings by showing that these regions are specifically associated with successful, rather than unsuccessful, imagery and retrieval performance, and also operate in a modality-independent fashion. As outlined in the discussion section, we interpret the activation in these regions as supporting the (re)construction and (re)experiencing of mental representations.

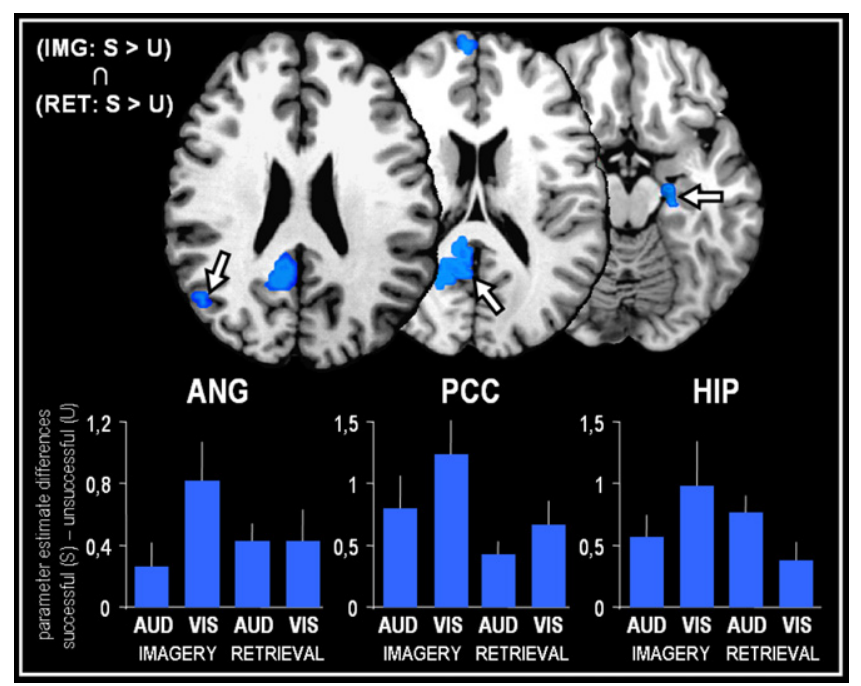

Fig. 2. Main positive effect: successful imagery and successful retrieval. Regions showing more activity for successful than unsuccessful trials regardless of task Bars reflect the mean activity (parameter estimates on the $y$-axis) for each region contrasting the difference between successful (S) vs. unsuccessful (U) imagery and successful (S) vs. unsuccessful (U) retrieval for both auditory (AUD) and visual (VIS) conditions. From left to right bars, show activity of the angular gyrus (ANG), posterior cingulate cortex (PCC) and hippocampus (HIP). Lines reflect the standard error of the mean.

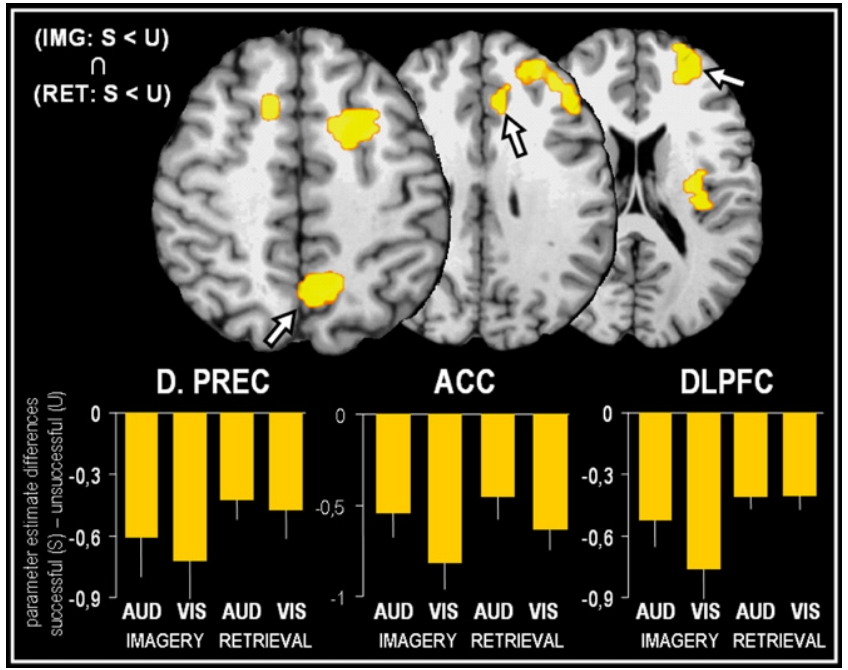

Fig. 3. Main negative effect: unsuccessful imagery and unsuccessful retrieval. Regions showing less activity for successful than unsuccessful trials regardless of task. Bars reflect the mean activity (parameter estimates on the $y$-axis) for each region contrasting the difference between successful (S) vs. unsuccessful (U) imagery and successful (S) vs. unsuccessful (U) retrieval for both auditory (AUD) and visual (VIS) conditions. From left to right bars, show activity of dorsal precuneus (D.PREC), anterior cingulate cortex (ACC) and dorsolateral prefrontal cortex (DLFPC). Lines reflect the standard error of the mean.

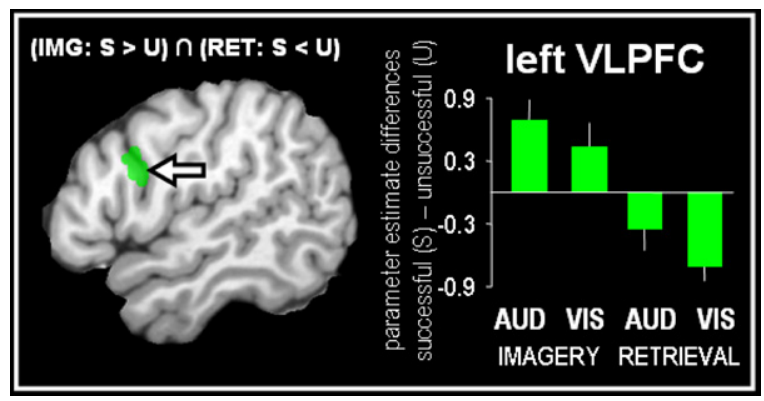

Fig. 4. Interaction I: successful imagery and unsuccessful retrieval. The left ventrolateral prefrontal cortex (VLPFC) shows greater activity for unsuccessful than successful retrieval trials, but greater activity for successful than unsuccessful imagery trials. Bars reflect the mean activity (parameter estimates on the $y$-axis) for the VLPFC contrasting the difference between successful (S) vs. unsuccessful (U) imagery and unsuccessful (U) vs. successful (S) retrieval for both auditory (AUD) and visual (VIS) conditions. Lines reflect the standard error of the mean. 
Table 4

Regions showing patterns of activity common to auditory and visual modalities.

\begin{tabular}{|c|c|c|c|c|c|c|c|c|c|c|c|c|c|c|c|c|c|}
\hline \multirow[t]{3}{*}{ Region } & \multirow[t]{3}{*}{ Side } & \multirow[t]{3}{*}{ BA } & \multicolumn{7}{|c|}{ Mental imagery } & \multicolumn{8}{|c|}{ Memory retrieval } \\
\hline & & & \multicolumn{3}{|c|}{ Max } & \multirow{2}{*}{$\begin{array}{l}\mathrm{S}>\mathrm{U} \\
\mathrm{T} \max \end{array}$} & \multirow[b]{2}{*}{ AUD } & \multirow[b]{2}{*}{ VIS } & \multirow{2}{*}{$\begin{array}{l}\text { Interaction } \\
\text { AUD > VIS }\end{array}$} & \multicolumn{4}{|l|}{$\operatorname{Max}$} & \multicolumn{3}{|l|}{$S>U$} & \multirow{2}{*}{$\begin{array}{l}\text { Interaction } \\
\text { AUD > VIS }\end{array}$} \\
\hline & & & $x$ & Y & $z$ & & & & & RT: $F>S$ & $x$ & Y & $Z$ & $\mathrm{~T} \max$ & AUD & VIS & \\
\hline \multicolumn{18}{|c|}{ Positive main effect: (IMG: $S>U$ ) and (RET: $S>U$ ) } \\
\hline Posterior cingulate cortex & $\mathrm{L}$ & $29 / 30$ & -6 & -51 & 12 & 4.63 & $\infty$ & $\infty$ & ns & na & -18 & -57 & 3 & 5.94 & 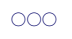 & $\infty$ & ns \\
\hline Medial prefrontal cortex & $\mathrm{L}$ & 10 & -3 & 66 & 9 & 3.54 & 0 & $\infty$ & ns & na & -3 & 63 & 6 & 3.41 & $\infty$ & 0 & ns \\
\hline Angular gyrus & $\mathrm{L}$ & 39 & -48 & -69 & 24 & 2.93 & + & $\infty$ & & na & -48 & -66 & 18 & 3.26 & $\infty$ & 0 & ns \\
\hline Hippocampus & $\mathrm{R}$ & na & 24 & -18 & -12 & 2.98 & $\infty$ & 0 & $\mathrm{~ns}$ & na & 24 & -21 & -18 & 4.80 & ০০০ & 0 & + \\
\hline Supplemental motor area & - & 6 & 0 & -12 & 54 & 4.32 & 0 & $\circ$ & ns & na & -3 & -15 & 48 & 4.59 & $\infty$ & $\infty$ & ns \\
\hline \multirow{2}{*}{$\begin{array}{l}\text { Putamen } \\
\text { Pulch }\end{array}$} & $\mathrm{L}$ & na & -18 & 9 & -15 & 5.62 & 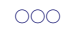 & ns & 0 & na & -21 & 6 & -15 & 5.40 & ০০০ & $\infty$ & ns \\
\hline & $\mathrm{R}$ & na & 15 & 9 & -12 & 4.98 & 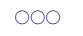 & + & ns & na & 18 & 12 & -12 & 5.47 & $\infty)^{-}$ & $\infty$ & ns \\
\hline \multicolumn{18}{|c|}{ Negative main effect: (IMG: $U>S)$ and $(R E T: U>S)$} \\
\hline \multicolumn{18}{|c|}{ RT sensitive } \\
\hline Anterior cingulate cortex & $\mathrm{R}$ & 32 & 9 & 30 & 30 & -6.35 & $\bullet \bullet \bullet$ & $\bullet \bullet \bullet$ & ns & $\bullet \bullet$ & 9 & 30 & 36 & -5.20 & $\bullet \bullet \bullet$ & $\bullet \bullet \bullet$ & ns \\
\hline Dorsal precuneus & $\mathrm{R}$ & 7 & 12 & -66 & 54 & -6.35 & $\bullet \bullet$ & $\bullet \bullet \bullet$ & ns & - & 9 & -63 & 48 & -6.32 & $\bullet \bullet \bullet$ & $\bullet \bullet$ & ns \\
\hline \multirow{2}{*}{\multicolumn{18}{|c|}{ RT insensitive }} \\
\hline & & & & & & & & & & & & & & & & & \\
\hline Dorsolateral prefrontal cortex & $\mathrm{R}$ & 46 & 33 & 51 & 18 & -7.49 & $\bullet \bullet \bullet$ & $\bullet \bullet \bullet$ & ns & ns & 30 & 42 & 18 & -6.48 & $\bullet \bullet \bullet$ & $\bullet \bullet \bullet$ & ns \\
\hline Superior prefrontal cortex & $\mathrm{R}$ & 9 & 42 & 27 & 39 & -5.33 & $\bullet \bullet$ & $\bullet \bullet \bullet$ & ns & ns & 42 & 30 & 33 & -5.46 & $\bullet \bullet \bullet$ & $\bullet \bullet \bullet$ & ns \\
\hline \multirow[t]{2}{*}{ Insula } & $\mathrm{L}$ & 13 & -48 & 12 & 3 & -3.73 & $\bullet \bullet \bullet$ & - & ns & ns & -48 & -18 & 0 & -3.24 & - & - & ns \\
\hline & $\mathrm{R}$ & 13 & 36 & -12 & 15 & -3.81 & $\bullet \bullet$ & $\bullet \bullet \bullet$ & ns & ns & 36 & -24 & 6 & -5.52 & - & $\bullet \bullet \bullet$ & ns \\
\hline \multicolumn{18}{|c|}{ Interaction I: (IMG: $S>U$ ) and (RET: $U>S$ ) } \\
\hline Ventrolateral prefrontal cortex & $\mathrm{L}$ & 45 & -54 & 6 & 21 & 4.24 & $\bigcirc$ & + & ns & na & -48 & 6 & 21 & -3.94 & - & $\bullet \bullet \bullet$ & ns \\
\hline \multicolumn{18}{|c|}{ Interaction II: (IMG: $U>S$ ) and (RET: $S>U$ ) } \\
\hline Ventral precuneus & $\mathrm{R}$ & 7 & 6 & -72 & 30 & -4.79 & $\bullet \bullet \bullet$ & - & ns & na & 9 & -66 & 27 & 4.12 & $\infty)^{-}$ & 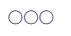 & ns \\
\hline Midcingulate cortex & $\mathrm{R}$ & $24 / 31$ & 9 & -36 & 39 & -6.25 & - & - & $\mathrm{ns}$ & na & 3 & -21 & 39 & 4.21 & 0 & 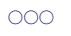 & $\mathrm{ns}$ \\
\hline Supramarginal gyrus & $\mathrm{R}$ & 40 & 48 & -51 & 51 & -3.67 & $\bullet \bullet$ & $\bullet$ & 0 & na & 48 & -48 & 45 & 5.99 & $\infty$ & $\infty$ & $\mathrm{ns}$ \\
\hline
\end{tabular}

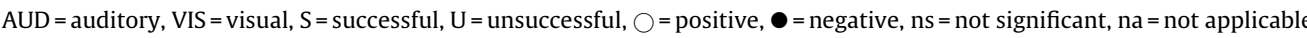
$P<0.10$ (trending).

$P<0.10$ (trending).

$P<0.05$.

$\bigcirc P<0.01$.

$000 P<0.001$. 


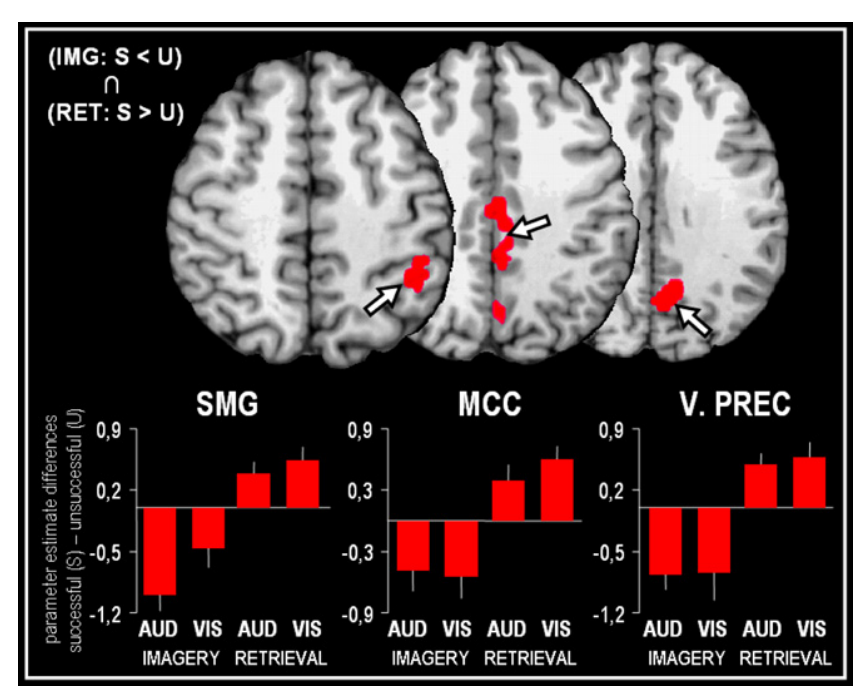

Fig. 5. Interaction II: unsuccessful imagery and successful retrieval. Regions showing greater activity for successful than unsuccessful retrieval trials, but greater activity for unsuccessful than successful imagery trials. Bars reflect the mean activity (parameter estimates on the $y$-axis) for each region contrasting the difference between unsuccessful (U) vs. successful (S) imagery and successful (S) vs. unsuccessful (U) retrieval for both auditory (AUD) and visual (VIS) conditions. From left to right bars, show activity of the supramarginal gyrus (SMG), midcingulate cortex (MCC) and ventral precuneus (V.PREC). Lines reflect the standard error of the mean. Lines reflect the standard error of the mean.

\subsubsection{Main negative effect: unsuccessful imagery and unsuccessful retrieval}

As shown in Fig. 3 and in line with our second prediction, the anterior cingulate cortex (ACC) and dorsolateral prefrontal cortex (DLPFC) showed greater activity for unsuccessful than successful imagery and retrieval, for both auditory and visual modalities. These findings are consistent with a recent study by Fleck, Daselaar, Dobbins, and Cabeza (2006) that compared successful and unsuccessful performance conditions during memory retrieval and a perceptual decision task. Similarly, they found increased activity in the ACC, DLPFC, as well as the dorsal precuneus, superior PFC and insula for unsuccessful performance conditions. Here, we not only confirm their pattern of results, but extend them to mental imagery. Outside of our predictions, but consistent with Fleck et al. (2006), the dorsal part of the precuneus also showed a pattern associated with unsuccessful performance regardless of modality. As outlined in the Introduction and Discussion sections, we interpret the activation in these regions as supporting effortful attentional control and monitoring processes.

\subsection{Interactions: task $\times$ performance}

Outside of our predictions, the left ventrolateral prefrontal cortex (VLPFC), showed a significant task $\times$ performance interaction showing greater activity for successful than unsuccessful imagery, but less activity for successful than unsuccessful retrieval (Fig. 4). As discussed later, we interpret this finding in relation to the role of this region in semantic memory processes. We also found regions that showed the opposite interaction, less activity for successful than unsuccessful imagery, but greater activity for successful than unsuccessful retrieval. As shown in Fig. 5 and Table 4, these regions involved the ventral precuneus, the midcingulate cortex (MCC), and right supramarginal gyrus. The finding of this pattern in ventral precuneus is very interesting, because it is one of the regions most consistently activated during tasks with a strong imagery component (Cavanna \& Trimble, 2006; Fletcher et al., 1995). Yet, as far as we know, this region has not yet been linked to poor imagery performance. As explained in the Introduction and Discussion sections, we interpret the activation in these regions as supporting mental inspection and working memory processes.

\subsection{Follow-up analyses}

We conducted four follow-up analyses. First, we tested to what extent brain regions that show similar activity patterns for auditory and visual conditions are driven by a single modality (Follow-up analysis 1, Supplemental Materials). As shown in Table 4 under the heading "A>V interaction", all regions - except for those within the parietal regions and the putamen - showed modalityindependent activity patterns. Secondly, we tested the influence of response times during the recognition judgments on regions showing a negative main effect (Follow-up analysis 2, Supplemental Materials). As shown in Table 4, the ACC and dorsal precuneus showed a significant effect of response time. These findings are consistent with Fleck et al. (2006) and will be discussed in relation to monitoring performance and cognitive control. Third, we assessed the influence of response times during the confidence rating on regions showing a negative main effect (Follow-up analysis 3, Supplemental Materials). As shown in Supplemental Fig. 1 and Supplemental Table 1, our findings cannot easily be attributed to response time differences in the rating procedures. Finally, we assessed the influence of low-confidence hits on the retrieval success effect (Follow-up analysis 4, Supplemental Materials). These results demonstrate that including low-confidence hits has little effect on the overall activity patterns, although it generally results in a slightly decreased activity difference between successful and unsuccessful performance, possibly due to guesses.

\section{Discussion}

As a first finding, we found that regions activated during auditory and visual perception are also activated during imagery and retrieval in a modality-specific fashion. As a second finding, we identified four sets of regions showing task (imagery/retrieval) $\times$ performance (successful/unsuccessful) main effects as well as interactions, which are modality-independent. A first set of regions, including hippocampus, posterior cingulate cortex (PCC), medial prefrontal cortex (mPFC) and angular gyrus, was associated with successful performance during both imagery and retrieval. A second set of regions, including the anterior cingulate cortex (ACC), superior/dorsolateral prefrontal cortex (DLPFC), dorsal precuneus, and insular cortex that is associated with unsuccessful performance in both tasks. A third region, the left ventrolateral prefrontal cortex (VLPFC) showed an interactions between task and performance for both auditory and visual modalities. A fourth set of regions, including ventral precuneus, the midcingulate cortex (MCC) and supramarginal gyrus, showed the opposite interaction between task and performance for both auditory and visual modalities. Below, we first discuss the role of the modality-specific regions in imagery and retrieval and then the modality-independent regions.

\subsection{Modality-specific effects: auditory and visual sensory regions contribute similarly to imagery and retrieval}

Leading models of imagery (Farah, 1984; Kosslyn et al., 1994) and retrieval (Marr, 1971; McClelland, McNaughton, \& O'Reilly, 1995; Tulving, 1983) assume that regions activated during realworld perception are also involved in the (re)construction of the sensory aspects of auditory and visual mental representations. These models have been supported by studies that examined imagery and retrieval or auditory and visual information separately. Imagery studies of auditory (Bunzeck, Wuestenberg, Lutz, Heinze, \&Jancke, 2005; Halpern \& Zatorre, 1999; Kosslyn, 2003) and 
visual information (Chen et al., 1998; Ishai, Ungerleider, \& Haxby, 2000; Kosslyn, 2003) found modality-specific activation in auditory and visual cortex respectively. Memory studies have found similar modality-specific involvement of auditory- and visual-processing regions (Nyberg et al., 2000; Wheeler et al., 2000a; Wheeler et al., $2000 \mathrm{~b}$ ). For instance, Wheeler and colleagues found that words accompanied by either auditory or visual information during learning also showed increased activity in auditory and visual cortices respectively when presented with those same words again during retrieval. In the current study, we confirmed the role auditory- and visual-processing regions in the construction of mental representations during imagery and retrieval. Yet, this is the first study that demonstrates the modality-specific role of auditory- and visualprocessing regions during both imagery and retrieval within the same experiment and the same participants.

\subsection{Modality-independent effects: Regions associated with successful imagery and successful retrieval}

The first set of regions included hippocampus, posterior cingulate cortex, medial prefrontal cortex and angular gyrus, and was associated both with imagery success (successful $>$ unsuccessful imagery) and retrieval success (successful > unsuccessful retrieval; Fig. 2). In line with several studies on episodic simulation of novel, future and past events, we interpret the activation overlap between imagery and retrieval as reflecting the (re)construction and (re)experiencing of mental representations derived from episodic memory (Addis et al., 2007; Hassabis et al., 2007a; Szpunar et al., 2007). The current study adds to these previous studies by showing that these regions are specifically linked to processes supporting successful imagery and retrieval. Below, we briefly discuss the possible role of these regions in reconstructive mental processes.

Main memory models assume that the hippocampus is involved in the formation of episodic memory traces, and plays a critical role in reactivation of memory traces during retrieval (Eichenbaum, Yonelinas, \& Ranganath, 2007; Squire, Stark, \& Clark, 2004). At the same time, models of mental imagery assume that information derived from episodic memory plays an important role in successful (re)construction of mental representations (Hassabis \& Maguire, 2007; Moulton \& Kosslyn, 2009). Strong support for a role of the hippocampus in imagery was provided by a recent study focusing on patients with hippocampal damage. In addition to severe episodic memory deficits, these patients show an impoverished ability to imagine fictitious events, even though these events never happened in their real lives (Hassabis, Kumaran, Vann, \& Maguire, 2007). These findings clearly illustrate that episodic processes play an important role in mental imagery regardless of whether events truly occurred in the past. Extrapolating these findings to our current results, we argue that the increase in hippocampal activity during successful imagery reflects the activation of episodic information, which contributes to the construction of a vivid and rich experience of both past and imaginary events.

The role of PCC in imagery and retrieval also confirms previous studies (Addis et al., 2007; Hassabis et al., 2007a; Szpunar et al., 2007) and can be explained by the strong anatomical (Kobayashi \& Amaral, 2003) and functional connections (Greicius, Supekar, Menon, \& Dougherty, 2009) of this region with the hippocampus. This region is among the areas most consistently activated during episodic retrieval tasks (Cabeza \& Nyberg, 2000) and lesions within the PCC can result in amnesic symptoms similar to those resulting from damage to the hippocampus (Valenstein et al., 1987). Here we confirm these findings under controlled memory conditions and show that imager- and retrieval-related activity in PCC occurs independent of sensory modality.
MPFC was also activated during successful imagery and retrieval of both auditory and visual information. This region tends to be activated during vivid retrieval (Daselaar, Fleck, \& Cabeza, 2006; Yonelinas, Otten, Shaw, \& Rugg, 2005) and during tasks that have a strong self-referential component (Buckner \& Carroll, 2007; Cabeza et al., 2004; Gusnard, Akbudak, Shulman, \& Raichle, 2001; Kelley et al., 2002). Although, our memory and imagery cues were not necessarily relating to ones self, we also found mPFC activity associated with successful imagery and retrieval in the current study. Again, the overlap between imagery and retrieval in mPFC suggests a supporting role of episodic memory processes in the construction of mental representations of familiar concepts, even when these are not necessarily referring to ones own personal past.

Despite the fact that the angular gyrus is one of the regions most consistently activated during successful retrieval, its role is still a matter of current debate (Wagner, Shannon, Kahn, \& Buckner, 2005). According to the episodic output buffer account, the angular gyrus forms a specialized memory buffer for episodic-like information possibly coming from the hippocampus (Vilberg \& Rugg, 2008; Wagner et al., 2005). This idea fits well with recent views that activity in the angular gyrus is sensitive to the amount and quality of information that is being generated in one's mind (Vilberg \& Rugg, 2008). Thus, in general the regional overlap between successful imagery and retrieval fits well with previous findings and current views about the functional role of these regions in memory.

\subsection{Modality-independent effects: Regions associated with unsuccessful imagery and unsuccessful retrieval}

The second set of regions including anterior cingulate cortex (ACC), dorsal precuneus, superior/dorsolateral prefrontal cortex (DLPFC) and bilateral insula showed activity associated with unsuccessful performance during both imagery and retrieval independent of sensory modality (Fig. 3). This pattern fits with a role of these regions in effortful imagery and retrieval processes associated with attention and monitoring. In terms of retrieval, successful retrieval conditions typically lead to faster responses made with greater confidence, whereas unsuccessful retrieval conditions lead to slower, low-confidence responses (e.g., Huijbers, Pennartz, Cabeza, \& Daselaar, 2009; Huijbers, Pennartz, \& Daselaar, 2009). Models of recognition memory assume that memory responses are based on the accumulation of evidence for or against a memory decision and the careful monitoring of retrieval output (Henson, Rugg, Shallice, \& Dolan, 2000; Henson, Shallice, \& Dolan, 1999; Ratcliff, 1978; Ratcliff \& Starns, 2009; Rugg \& Wilding, 2000; Tulving, 1983). When the decision is more difficult more evidence needs to be accumulated leading to slower response times. In line with a general role in effortful decision making, the regions activated during unsuccessful retrieval have also been linked to effortful decisions outside the memory domain. For instance, Fleck et al. (2006) found that ACC and DLPFC activity was not specific to retrieval effort, but also correlated with effort during a perceptual decision task (Fleck et al., 2006).

We also conducted a follow-up response time analysis focusing only on the successful retrieval trials. This analysis revealed that ACC and dorsal precuneus also showed greater activity during slow as compared to fast correct memory trials, whereas the superior/dorsolateral PFC and the insula did not. These results generally confirm previous findings by Fleck and colleagues who separated the contributions of decision confidence and reaction times to ACC and DLPFC activity during both a retrieval task and a perceptual decision task (Fleck et al., 2006). Whereas the ACC was associated more with slow response times, the DLPFC was associated more with low levels of confidence. Based on these results, Fleck et al. concluded that the ACC is involved in continuous conflict monitoring, whereas the DLPFC is involved in the evaluation of accumulated 
information and response selection. Although they focused their analyses on the ACC and DLPFC, the dorsal precuneus and insula were also associated with effortful processes during both memory retrieval and perception. As noted, the current results extend these memory and perception findings by suggesting a similar involvement of these regions in mental imagery.

With respect to the specific role of the ACC, dorsal precuneus and superior PFC/DLPFC, each of these regions have been linked to attentional processes involved in the careful monitoring and evaluation of information activated during the search for a memory trace (Fleck et al., 2006; Henson, Rugg, Shallice, Josephs, \& Dolan, 1999; Kim \& Cabeza, 2009). Moreover, the perception task used in the study by Fleck et al. involved a complex size judgment, which also required careful evaluation of alternative options. Similar attentional processes have been suggested to play an important role in mental imagery by helping to initiate, inspect and terminate the imagery process when a specific goal has been met (Moulton \& Kosslyn, 2009). The ACC and DLFPC have repeatedly been linked to top-down attention mechanisms necessary for monitoring performance and cognitive control (Egner \& Hirsch, 2005; MacDonald, Cohen, Stenger, \& Carter, 2000). Also, the dorsal part of the precuneus is believed to support goal-directed imagery processes, and has been linked to selection of relevant details during mental imagery (Ganis, Thompson, \& Kosslyn, 2004). Finally, superior PFC has been strongly linked to a frontoparietal top-down attention system (Corbetta \& Shulman, 2002; Corbetta, Patel, \& Shulman, 2008). Thus, the current findings linking these regions to general effort fit well with previous findings. At the same time, we believe that the insular cortex is not part of the attention/monitoring set of regions. Activity in this region has been linked to performance failure in various tasks (Daselaar, Prince, \& Cabeza, 2004; Menon, Adleman, White, Glover, \& Reiss, 2001), as well as to elevated levels of stress and arousal (Liotti et al., 2000; Paulus, Rogalsky, Simmons, Feinstein, \& Stein, 2003). Thus, one possible explanation for this last finding is that insular activity during imagery and retrieval reflects high levels of arousal or stress associated with performance failure. Finally, we should note that, even though our behavioral and fMRI findings fit well with previous studies, we cannot exclude the possibility that some of the activations we found for the unsuccessful vs. successful performance comparisons, reflect either inattention to or distraction from the stimuli and task.

\subsection{Modality-independent effects: Regions showing interaction between task and performance}

We also found interactions between mental imagery and memory retrieval. The left ventrolateral prefrontal cortex (VLPFC) showed greater activity during successful imagery than successful retrieval (Fig. 4). The left VLPFC is strongly activated during semantic classification and production tasks (Grossman et al., 2002; Thompson, D’Esposito, Aguirre, \& Farah, 1997; Vandenberghe, Price, Wise, Josephs, \& Frackowiak, 1996). One notable difference between the tasks is that the imagery condition uses a novel cue word describing a semantic entity, whereas the retrieval task uses a previously studied cue. Thus compared to episodic retrieval, successful mental imagery will rely more heavily on semantic generation and elaboration processes to construct a mental representation based on a novel imagery cue (Addis et al., 2007; Kosslyn et al., 2001). A similar semantic generation strategy can be used during retrieval. However, this will be particularly the case when the memory cue does not automatically activate an appropriate memory trace, and the retrieval search is extended and likely to fail, which might explain the pattern we found in this region. Yet, the current data do not allow any strong conclusions regarding the role of VLPFC in imagery and retrieval.
Ventral precuneus, the midcingulate cortex and supramarginal gyrus were associated with successful retrieval performance but with unsuccessful imagery performance (Fig. 5). The finding that ventral precuneus is associated with unsuccessful imagery is surprising. Ventral precuneus is one of the regions most consistently activated during tasks that are heavily dependent on visual imagery (Fletcher et al., 1995; Kosslyn, 2003). For this reason, this region has even been dubbed "the mind's eye" (Fletcher et al., 1995; Kosslyn, 2003). Here, we confirm the involvement of ventral precuneus in visual imagery, but our findings indicate that this region plays a similar role in auditory imagery. Moreover, our data suggest that activity in this region is not linked to successful imagery processes, but rather to processes associated with unsuccessful imagery.

One interpretation for the interaction between unsuccessful imagery and successful retrieval is provided by models of imagery that include mental inspection and working memory processes (Farah, 1984; Kosslyn et al., 1994; Moulton \& Kosslyn, 2009). These models state that during imagery, information first has to become accessible within working memory, before it can be mentally inspected. Similar to more semantic generation during unsuccessful retrieval, relatively unsuccessful imagery may lead to the generation of more alternative mental representations that need to be selected and held in working memory as compared to a successful imagery event.

A working memory interpretation fits well with evidence that ventral precuneus, the midcingulate cortex and supramarginal gyrus have all been linked to working memory maintenance processes (Cohen et al., 1997; Petit, Courtney, Ungerleider, \& Haxby, 1998; Schon, Quiroz, Hasselmo, \& Stern, 2009). Moreover, these regions are activated during tasks that tax working memory demands, such as mental arithmetics, deductive reasoning and visuospatial planning (Knauff, Mulack, Kassubek, Salih, \& Greenlee, 2002; Menon, Rivera, White, Glover, \& Reiss, 2000; van den Heuvel et al., 2003). Damage to these regions results in a condition called dyscalculia involving deficits in mental arithmetics (Jung et al., 2001), as well as in impoverished autobiographical memories, which lack in explicit perceptual details (Berryhill, Phuong, Picasso, Cabeza, \& Olson, 2007). These patient findings could easily be accounted for by a working memory deficit. Thus, we speculate that the supramarginal gyrus, midcingulate cortex and ventral precuneus assist in the mental inspection of alternative mental representations, and that this mental process is closely linked to working memory. At the same time, we should acknowledge that other interpretations of the imagery and retrieval activations in these regions are possible. For instance, even though the follow-up response time analysis does not seem to support this explanation (see Supplemental Materials), the interaction between imagery (unsuccessful > successful) and retrieval (successful> unsuccessful) may still be related to the different types of ratings (quality vs. confidence) in the two tasks. One explanation for the opposite effect during retrieval - greater activity during successful vs. unsuccessful retrieval - is that successful retrieval leads to the generation of episodic information, which needs to be maintained in working memory, whereas unsuccessful retrieval may not. Future research will be necessary to further separate the neural and cognitive components of unsuccessful memory processes, in particular, the difference between multiple attempts vs. a failure to imagine/retrieve information.

\section{Conclusions}

In this fMRI study, we contrasted the neural correlates of successful and unsuccessful mental imagery and memory retrieval. We identified four distinct patterns of activity in several brain regions, which were shared by auditory and visual modalities. The first set of regions - including hippocampus posterior cingulate cortex, 
medial prefrontal cortex and angular gyrus - was associated with successful performance regardless of task. This pattern is consistent with the conscious experiencing of past and imaginary events. The second set of regions - including dorsal precuneus, anterior cingulate and dorsolateral prefrontal cortex - showed the opposite pattern, again common to imagery and retrieval and consistent with attention/monitoring processes. Third, the left VLPFC showed an interaction between task (imagery/retrieval) and performance (successful/unsuccessful) This pattern is consistent with semantic generation processes, that might assist mental imagery when episodic retrieval is unsuccessful. The final set of regions - including ventral precuneus, midcingulate cortex and supramarginal gyrus showed the opposite interaction between task and performance. This pattern is consistent with components of working memory that benefit retrieval and imagery. This is the first study to separate the neural correlates of successful and unsuccessful performance for both imagery and retrieval.

\section{Methods}

\subsubsection{Participants}

Twenty-one participants ( 16 female, mean age 22 ) recruited from the University of Amsterdam community took part in the experiment. All participants were in good health, and right-handed. Their native language was Dutch and they were paid 65 euro for participation. All participants gave their informed consent and the study met the criteria for approval of the Academic Medical Center Medical Ethical Committee.

\subsubsection{Stimul}

The stimuli consisted of 456 Dutch cue-words (nouns) specifically matched with 456 corresponding images and 456 corresponding sounds. The matched sounds were 2-channel stereo with a sample rate of $22 \mathrm{kHz}, 16$-bit sample size, WAV-format, with a duration of $3 \mathrm{~s}$. The matched images consisted of $640 \times 480$ pixels, 16 -bit color, BMP-format and were also presented for $3 \mathrm{~s}$. To mimic the dynamical characteristic of sounds, the images faded-in ( $1 \mathrm{~s})$, stayed on the screen ( $1 \mathrm{~s})$ and then faded-out (1 s). Visual stimuli were projected on a screen at the foot of the fMRI scanner, and seen via a mirror mounted on the head-coil. Auditory stimuli were presented via a MR-compatible headphone with passive noise dampening (MR Confon). Behavioral responses were collected by an MR-compatible four-button box (Lumitouch).

\subsubsection{Procedure}

Before the experiment, cue-words were randomly assigned to one of four conditions for each individual subject: (1) imagine sound, (2) hear sound, (3) imagine image or (4) observe image. The cue-words were carefully selected so they could match each condition. Thus, for example, cue-words like "cat" or "airplane" can easily be presented or imagined as either a sound or visual image. Before the start of the actual experiment, participants conducted a brief 20-trial practice session to check the volume of the headphones and to habituate to the scanner noise. The presentation order of the stimuli was pseudorandomized, to ensure that two consecutive trials were never of the same condition. Because pilot studies had indicated that the "imagine sound" condition was slightly more difficult and to ensure a sufficient number of successful trials in each of the four conditions, we used 132 trials for the "imagine sound" condition and 108 trials for the other conditions.

As shown in Table 1, the fMRI experiment consisted of an imagery task and a retrieval task on consecutive days. Each task consisted of six runs, approximately $8 \mathrm{~min}$ long and 76 trials each. During the imagery task, a single trial consisted of a cue period, an imagery/perception period, and a rating period. During the cue period, a 1000-ms cue-word was presented with an instruction to imagine or perceive a sound or image associated with the cue word. During the imagery/perception period, participants either imagined or perceived an image or sound according to the instruction. During the $1500-\mathrm{ms}$ rating period, participants rated on a fourpoint scale ( 1 = very low, 4 = very high) the quality of their imagery experience or the richness of their perceptual experience. Perceptual richness was defined as the amount of subjectively experienced perceptual detail. Critically, participants were not informed that their memory for these events would be tested the next day during the retrieval task. A retrieval trial consisted of a retrieval period and a rating period. During the 4000-ms retrieval period, the cue-words from the previous day were presented again and participants indicated the condition in which they had seen the word the day before (imagined sound/heard sound/imagined image/observed image). During the 1500-ms rating period, participants rated the confidence of their judgment on a two-point scale (unsure/sure). During both imagery/perception and memory retrieval phases, the intertrial intervals were jittered between 100 and $2100 \mathrm{~ms}$. As illustrated in Table 1, analyses included both mental imagery and perceptual data during the first day, but in keeping with previous fMRI studies that compared the neural correlates of imagery and retrieval (e.g. Buckner \&
Wheeler, 2001; Cabeza, Locantore, \& Anderson, 2003; Prince, Daselaar, \& Cabeza 2005; Spaniol et al., 2009), we only analyzed retrieval of perceived auditory and visual events.

\subsubsection{Scanning parameters}

Functional MRI images were collected on a Phillips Intera 3.0T using a 6-channel SENSE head coil and a T2* sensitive gradient echo sequence $(96 \times 96$ matrix, TR $2000 \mathrm{~ms}$, TE $30 \mathrm{~ms}$, FA $80^{\circ}, 34$ slices, $2.3 \mathrm{~mm} \times 2.3 \mathrm{~mm}$ voxel size, $3-\mathrm{mm}$ thick transverse slices). Additionally, a high-resolution T1-weighted structural scan $(256 \times 256$ matrix, TR $12 \mathrm{~ms}$, TE 5ms, FOV $24 \mathrm{~cm}, 68$ slices, $1 \mathrm{~mm}$ slice thickness) was collected on the second day.

\subsection{5. fMRI preprocessing and analysis}

Statistical Parametric Mapping (SPM5; (http://www.fil.ion.ucl.ac.uk/spm) software was used to preprocess and analyze the MR data. For each session, functional images were slice-time corrected, motion-corrected, coregistered to the structural scan acquired during the second day, and then normalized. First, individual nor malization parameters were obtained by normalizing the segmented structural scan of each subject using the Montreal Neurological Institute (MNI) T1 template image. These normalization parameters were then applied to the functional images. Next, the normalized functional images were resliced to a resolution of $3 \mathrm{~mm} \times 3 \mathrm{~mm} \times 3 \mathrm{~mm}$ and spatially smoothed using an $8-\mathrm{mm}$ isotropic Gaussian kernel. For each subject, trial related activity was modeled by convolving a vector of trial onsets with a canonical hemodynamic response function (HRF). The General Linear Model (GLM), as implemented in SPM5, was used to model effects of interest and remove confounding effects. Statistical Parametrical Maps were identified for each participant by applying linear contrasts to the parameter estimates (beta weight) for the events of interest, resulting in a $t$-statistic for every voxel. Group effects were assessed by applying random effects analyses.

We distinguished between 12 relevant trial types: low and high perception, unsuccessful and successful imagery, and unsuccessful and successful retrieval of perceived items, for both auditory and visual trials. We defined low perception as rating 1 and 2 on the four-point quality/richness scale and high perception as rating 4. Similarly, we defined unsuccessful imagery as rating 1 and 2 on the four-point quality/richness scale, and successful imagery as rating 4 . We defined successful memory as the correct responses that were rated as confident, while unsuccessful memory was defined as all incorrect responses regardless of confidence. We also modeled successful and unsuccessful retrieval of previously imagined information separately, but we do not report this data here (see Table 2). Other trials, including omitted responses, were included as a separate trial type of no interest.

\subsubsection{Modality-specific activation of sensory regions}

To assess whether, in line with previous fMRI studies, activity in sensory regions was associated with imagery and retrieval in a modality-specific fashion (Buckner \& Wheeler, 2001; Kosslyn et al., 2001; Kraemer et al., 2005; Shannon \& Buckner, 2004), we used a four-step approach for each modality separately. For the auditory modality, we first compared the externally presented items in the visual modality to those presented in the auditory modality (high auditory perception $>$ high visual perception). Second, we identified regions related to auditory mental imagery (successful auditory imagery > successful visual imagery). Third, we performed the same step for auditory memory retrieval (successful auditory retrieval > successful visual retrieval). Fourth, we inclusively masked the resulting T-maps to look for overlaps between auditory perception, auditory imagery and auditory retrieval. For each map, we used an uncorrected threshold of $P<0.023$ with a cluster size of 8 for each map. Given that perception, imagery and retrieval were measured during independent trials, the joint probability for three independent measures can be calculated using the Fisher's method, resulting in a joint probability of $P<0.001$ (Mosteller \& Fisher, 1948). The same four steps were repeated for the visual modality.

\subsubsection{Activation of modality-independent regions}

To assess overlap in activations of modality-independent regions involved in imagery and retrieval, we combined the auditory and visual trials. Next, we crossed the factors task (memory/imagery) and performance (successful/unsuccessful) and created maps using inclusive masking (also at $P<0.01$ uncorrected, $c=8$ ) for all four combinations (1. [successful $>$ unsuccessful retrieval] $\cap$ [successful $>$ unsuccessful imagery]; 2. [unsuccessful $>$ successful retrieval] $\cap[$ unsuccessful $>$ successful imagery]; 3. [successful $>$ unsuccessful retrieval] $\cap$ [unsuccessful $>$ successful imagery]; 4. [unsuccessful $>$ successful retrieval] $\cap[$ successful $>$ unsuccessful imagery]). Given that imagery and retrieval were measured during independent trials, the joint probability can again be calculated using the Fisher's method, resulting also in a joint probability of $P<0.001$ (Mosteller \& Fisher, 1948). To assess whether the regions identified showed an activation pattern consistent with a modality-independent role, we extracted their mean cluster activity and checked whether the patterns were significant for both modalities at $P<0.05$, uncorrected. 


\section{Acknowledgements}

The authors sincerely thank the reviewer for providing constructive comments. Funding: WH. was supported by the Amsterdam Brain Imaging Platform (ABIP), CMP. by the Netherlands Organization for Scientific Research (NWO) grant 918.46.609 and SMD. by NWO grant 916.66 .022 and ABIP.

\section{Appendix A. Supplementary data}

Supplementary data associated with this article can be found, in the online version, at doi:10.1016/j.neuropsychologia.2011.02.051.

\section{References}

Addis, D. R., Pan, L., Vu, M. A., Laiser, N., \& Schacter, D. L. (2009). Constructive episodic simulation of the future and the past: Distinct subsystems of a core brain network mediate imagining and remembering. Neuropsychologia, 47(11), 2222-2238.

Addis, D. R., Wong, A. T., \& Schacter, D. L. (2007). Remembering the past and imagining the future: Common and distinct neural substrates during event construction and elaboration. Neuropsychologia, 45(7), 1363-1377.

Baddeley, A. (1992). What is autobiographical memory? In C. M. A. D. C. Rubin, H. Spinnler, \& W. A. Wagenaar (Eds.), Theoretical perspectives on autobiographical memory (pp. 13-29). Dordrecht: Kluwer.

Bartlett, F. C. (1932). Remembering. Cambridge, UP: Cambridge.

Berryhill, M. E., Phuong, L., Picasso, L., Cabeza, R., \& Olson, I. R. (2007). Parietal lobe and episodic memory: Bilateral damage causes impaired free recall of autobiographical memory. Journal of Neuroscience, 27(52), 14415-14423.

Buckner, R. L., \& Carroll, D. C. (2007). Self-projection and the brain. Trends in Cognitive Sciences, 11(2), 49-57.

Buckner, R. L., Logan, J., Donaldson, D. I., \& Wheeler, M. E. (2000). Cognitive neuroscience of episodic memory encoding. Acta Psychologica, 105(2-3), 127-139.

Buckner, R. L., \& Wheeler, M. E. (2001). The cognitive neuroscience of remembering. Nature Reviews Neuroscience, 2(9), 624-634.

Bunzeck, N., Wuestenberg, T., Lutz, K., Heinze, H.-J., \& Jancke, L. (2005). Scanning silence: Mental imagery of complex sounds. Neuroimage, 26(4), 1119-1127.

Cabeza, R., Locantore, J. K., \& Anderson, N. D. (2003). Lateralization of prefrontal activity during episodic memory retrieval: Evidence for the production-monitoring hypothesis. Journal of Cognitive Neuroscience, 15(2), 249-259.

Cabeza, R., \& Nyberg, L. (2000). Imaging cognition II: An empirical review of 275 PET and fMRI studies. Journal of Cognitive Neuroscience, 12(1), 1-47.

Cabeza, R., Prince, S. E., Daselaar, S. M., Greenberg, D. L., Budde, M., Dolcos, F., et al. (2004). Brain activity during episodic retrieval of autobiographical and laboratory events: An fMRI study using a novel photo paradigm. Journal of Cognitive Neuroscience, 16(9), 1583-1594.

Cabeza, R., \& St Jacques, P. (2007). Functional neuroimaging of autobiographical memory. Trends in Cognitive Sciences, 11(5), 219-227.

Cavanna, A. E., \& Trimble, M. R. (2006). The precuneus: A review of its functional anatomy and behavioural correlates. Brain, 129(Pt 3), 564-583.

Chen, W., Kato, T., Zhu, X.-H., Ogawa, S., Tank, D. W., \& Ugurbil, K. (1998). Human primary visual cortex and lateral geniculate nucleus activation during visua imagery. Neuroreport, 9(16), 3669-3674.

Cohen, J. D., Perlstein, W. M., Braver, T. S., Nystrom, L. E., Noll, D. C., Jonides, J., et al. (1997). Temporal dynamics of brain activation during a working memory task. Nature, 386(6625), 604-608.

Corbetta, M., \& Shulman, G. L. (2002). Control of goal-directed and stimulus-driven attention in the brain. Nature Reviews Neuroscience, 3(3), 201-215.

Corbetta, M., Patel, G., \& Shulman, G. L. (2008). The reorienting system of the human brain: from environment to theory of mind. Neuron, 58(3), 306-324.

Daselaar, S. M., Fleck, M. S., \& Cabeza, R. (2006). Triple dissociation in the medial temporal lobes: Recollection, familiarity, and novelty. Journal of Neurophysiology, 96(4), 1902-1911.

Daselaar, S. M., Prince, S. E., \& Cabeza, R. (2004). When less means more: Deactivations during encoding that predict subsequent memory. Neuroimage, 23(3), 921-927.

Daselaar, S. M., Rice, H.J., Greenberg, D. L., Cabeza, R., LaBar, K. S., \& Rubin, D. C. (2008). The spatiotemporal dynamics of autobiographical memory: Neural correlates of recall, emotional intensity, and reliving. Cerebral Cortex, 18(1), 217-229.

Deacon, T., Kosslyn, S. M., \& Scarry, E. (2001). Science, culture, meaning, values: A dialogue. Annals of New York Academy Science, 935, 233-257.

Egner, T., \& Hirsch, J. (2005). Cognitive control mechanisms resolve conflict through cortical amplification of task-relevant information. Nature Neuroscience, 8(12), 1784-1790.

Eichenbaum, H., Yonelinas, A. P., \& Ranganath, C. (2007). The medial temporal lobe and recognition memory. Annual Reviews Neuroscience, 30, 123-152.

Farah, M. J. (1984). The neurological basis of mental imagery: A componential analysis. Cognition, 18(1-3), 245-272.

Fleck, M. S., Daselaar, S. M., Dobbins, I. G., \& Cabeza, R. (2006). Role of prefrontal and anterior cingulate regions in decision-making processes shared by memory and nonmemory tasks. Cerebral Cortex,
Fletcher, P. C., Frith, C. D., Baker, S. C., Shallice, T., Frackowiak, R. J., \& Dolan, R.J.(1995), The minds eye-precuneus activation in memory-related imagery. Neuroimage, 2(3), 195-200.

Ganis, G., Thompson, W. L., \& Kosslyn, S. M. (2004). Brain areas underlying visual mental imagery and visual perception: An fMRI study. Brain Research Cognitive Brain Research, 20(2), 226-241.

Greenberg, D. L., \& Rubin, D. C. (2003). The neuropsychology of autobiographical memory. Cortex, 39(4-5), 687-728.

Greicius, M. D., Supekar, K., Menon, V., \& Dougherty, R. F. (2009). Resting-state functional connectivity reflects structural connectivity in the default mode network Cerebral Cortex, 19(1), 72-78.

Grossman, M., Koenig, P., DeVita, C., Glosser, G., Alsop, D., Detre, J., et al. (2002). The neural basis for category-specific knowledge: An fMRI study. Neuroimage, 15(4), 936-948.

Gusnard, D. A., Akbudak, E., Shulman, G. L., \& Raichle, M. E. (2001). Medial prefrontal cortex and self-referential mental activity: Relation to a default mode of brain function. Proceedings of the National Academy of Sciences of the United States of America, 98(7), 4259-4264.

Halpern, A. R., \& Zatorre, R. J. (1999). When that tune runs through your head: A PET investigation of auditory imagery for familiar melodies. Cerebral Cortex, 9(7), 697-704.

Hassabis, D., Kumaran, D., \& Maguire, E. A. (2007). Using imagination to understand the neural basis of episodic memory. Journal of Neuroscience, 27(52), $14365-14374$.

Hassabis, D., Kumaran, D., Vann, S. D., \& Maguire, E. A. (2007). Patients with hippocampal amnesia cannot imagine new experiences. Proceedings of the National Academy of Sciences of the United States of America, 104(5), 1726-1731.

Hassabis, D., \& Maguire, E. A. (2007). Deconstructing episodic memory with construction. Trends in Cognitive Sciences, 11(7), 299-306.

Henson, R., Rugg, M., Shallice, T., \& Dolan, R. (2000). Confidence in recognition memory for words. Journal of Cognitive Neuroscience, 23-123.

Henson, R. A., Rugg, M. D., Shallice, T., Josephs, O., \& Dolan, R. J. (1999). Recollection and familiarity in recognition memory: An event-related functional magnetic resonance imaging study. Journal of Neuroscience, 19(10), 3962-3972.

Henson, R. A., Shallice, T., \& Dolan, R. J. (1999). Right prefrontal cortex and episodic memory retrieval: A functional MRI test of the monitoring hypothesis. Brain, 122(Pt 7), 1367-1381.

Huijbers, W., Pennartz, C. M., Cabeza, R., \& Daselaar, S. M. (2009). When learning and remembering compete: A functional MRI study. PLoS Biology, 7(1), e11.

Huijbers, W., Pennartz, C. M., \& Daselaar, S. M. (2009). Dissociating the "retrieval success" regions of the brain: Effects of retrieval delay. Neuropsychologia.

Ishai, A., Ungerleider, L. G., \& Haxby, J. V. (2000). Distributed neural systems for the generation of visual images. Neuron, 28(3), 979-990.

Jung, R. E., Yeo, R. A., Sibbitt, W. L., Jr., Ford, C. C., Hart, B. L., \& Brooks, W. M. (2001). Gerstmann syndrome in systemic lupus erythematosus: Neuropsychological, neuroimaging and spectroscopic findings. Neurocase, 7(6), 515-521.

Kelley, W. M., Macrae, C. N., Wyland, C. L., Caglar, S., Inati, S., \& Heatherton, T. F. (2002). Finding the self? An event-related fMRI study. Journal of Cognitive Neuroscience, 14(5), 785-794.

Kim, H., \& Cabeza, R. (2009). Common and specific brain regions in high- versus low-confidence recognition memory. Brain Research, 1282, 103-113.

Knauff, M., Mulack, T., Kassubek, J., Salih, H. R., \& Greenlee, M. W. (2002). Spatial imagery in deductive reasoning: A functional MRI study. Brain Research Cognitive Brain Research, 13(2), 203-212.

Kobayashi, Y., \& Amaral, D. G. (2003). Macaque monkey retrosplenial cortex. II Cortical afferents. Journal of Comparative Neurology, 466(1), 48-79.

Kosslyn, S. M. (2003). Understanding the mind's eye and nose. Nature Neuroscience, 6(11), 1124-1125.

Kosslyn, S. M., Alpert, N. M., Thompson, W. L., Chabris, C. F., Rauch, S. L., \& Anderson, A. K. (1994). Identifying objects seen from different viewpoints, a PET investigation. Brain, 117(Pt 5), 1055-1071.

Kosslyn, S. M., Ganis, G., \& Thompson, W. L. (2001). Neural foundations of imagery. Nature Reviews Neuroscience, 2(9), 635-642.

Kraemer, D. J., Macrae, C. N., Green, A. E., \& Kelley, W. M. (2005). Musical imagery: Sound of silence activates auditory cortex. Nature, 434(7030), 158.

Liotti, M., Mayberg, H. S., Brannan, S. K., McGinnis, S., Jerabek, P., \& Fox, P. T. (2000). Differential limbic-cortical correlates of sadness and anxiety in healthy subjects: Implications for affective disorders. Biological Psychiatry, 48(1), 30-42.

Marr, D. (1971). Simple memory: A theory for archicortex. Philosophical Transactions of the Royal Society of London Series B: Biological Sciences, 262(841), 23-81.

McClelland, J. L., McNaughton, B. L., \& O'Reilly, R. C. (1995). Why there are complementary learning systems in the hippocampus and neocortex: Insights from the successes and failures of connectionist models of learning and memory. Psychological Review, 102(3), 419-457.

MacDonald, A. W., III, Cohen, J. D., Stenger, V. A., \& Carter, C. S. (2000). Dissociating the role of the dorsolateral prefrontal and anterior cingulate cortex in cognitive control. Science, 288(5472), 1835-1838.

Menon, V., Adleman, N. E., White, C. D., Glover, G. H., \& Reiss, A. L. (2001). Errorrelated brain activation during a Go/NoGo response inhibition task. Human Brain Mapping, 12(3), 131-143.

Menon, V., Rivera, S. M., White, C. D., Glover, G. H., \& Reiss, A. L. (2000). Dissociating prefrontal and parietal cortex activation during arithmetic processing. Neuroimage, 12(4), 357-365.

Mosteller, F., \& Fisher, R. A. (1948). Questions and answers. The American Statistician, 2(5), 30-31. 
Moulton, S. T., \& Kosslyn, S. M. (2009). Imagining predictions: Mental imagery as mental emulation. Philosophical Transactions of the Royal Society of London Series B: Biological Sciences, 364(1521), 1273-1280.

Nyberg, L., Habib, R., Mcintosh, A. R., \& Tulving, E. (2000). Reactivation of encodingrelated brain activity during memory retrieval. Proceedings of the National Academy of Sciences of the United States of America, 97(20), 11120-11124.

Paulus, M. P., Rogalsky, C., Simmons, A., Feinstein, J. S., \& Stein, M. B. (2003). Increased activation in the right insula during risk-taking decision making is related to harm avoidance and neuroticism. Neuroimage, 19(4), 1439-1448.

Petit, L., Courtney, S. M., Ungerleider, L. G., \& Haxby, J. V. (1998). Sustained activity in the medial wall during working memory delays. Journal of Neuroscience, 18(22), 9429-9437.

Prince, S. E., Daselaar, S. M., \& Cabeza, R. (2005). Neural correlates of relational memory: Successful encoding and retrieval of semantic and perceptual associations. Journal of Neuroscience, 25(5), 1203-1210.

Ratcliff, R. (1978). A theory of memory retrieval [review]. Psychological Review, 88(88), 552-572.

Ratcliff, R., \& Starns, J. J. (2009). Modeling confidence and response time in recognition memory. Psychological Review, 116(1), 59-83.

Rubin, D. C., Schrauf, R. W., \& Greenberg, D. L. (2003). Belief and recollection of autobiographical memories. Memory \& Cognition, 31(6), 887-901.

Rugg, M. D., \& Wilding, E. L. (2000). Retrieval processing and episodic memory. Trends in Cognitive Sciences, 4(3), 108-115.

Schacter, D. L., Addis, D. R., \& Buckner, R. L. (2007). Remembering the past to imagine the future: The prospective brain. Nature Reviews Neuroscience, 8(9), 657-661.

Schon, K., Quiroz, Y. T., Hasselmo, M. E., \& Stern, C. E. (2009). Greater working memory load results in greater medial temporal activity at retrieval. Cerebral Cortex, 19(11), 2561-2571.

Shannon, B. J., \& Buckner, R. L. (2004). Functional-anatomic correlates of memory retrieval that suggest nontraditional processing roles for multiple distinct regions within posterior parietal cortex. Journal of Neuroscience, 24(45), 10084-10092.

Spaniol, J., Davidson, P. S., Kim, A. S., Han, H., Moscovitch, M., \& Grady, C. L. (2009). Event-related fMRI studies of episodic encoding and retrieval: Meta-analyses using activation likelihood estimation. Neuropsychologia, 47(8-9), 1765-1779.
Squire, L. R., Stark, C. E., \& Clark, R. E. (2004). The medial temporal lobe. Annua Reviews Neuroscience, 27, 279-306.

Szpunar, K. K., Watson, J. M., \& McDermott, K. B. (2007). Neural substrates of envisioning the future. Proceedings of the National Academy of Sciences of the United States of America, 104(2), 642-647.

Thompson, S. S. L., D’Esposito, M., Aguirre, G. K., \& Farah, M. J. (1997). Role of left inferior prefrontal cortex in retrieval of semantic knowledge: A reevaluation. Proceedings of the National Academy of Sciences of the United States of America, 94(26), 14792-14797.

Tulving, E. (1983). Elements of episodic memory. Oxford: Clarendon Press.

Valenstein, E., Bowers, D., Verfaellie, M., Heilman, K. M., Day, A., \& Watson, R. T. (1987). Retrosplenial amnesia. Brain, 110(Pt 6), 1631-1646.

van den Heuvel, O. A., Groenewegen, H. J., Barkhof, F., Lazeron, R. H., van Dyck, R., \& Veltman, D. J. (2003). Frontostriatal system in planning complexity: A parametric functional magnetic resonance version of Tower of London task. Neuroimage, 18(2), 367-374.

Vandenberghe, R., Price, C., Wise, R., Josephs, O., \& Frackowiak, R. S. J. (1996). Functional anatomy of a common semantic system for words and pictures. Nature, 383(6597), 254-256.

Vilberg, K. L., \& Rugg, M. D. (2008). Memory retrieval and the parietal cortex: A review of evidence from a dual-process perspective. Neuropsychologia, 46(7), 1787-1799.

Wagner, A. D., Shannon, B. J., Kahn, I., \& Buckner, R. L. (2005). Parietal lobe contributions to episodic memory retrieval. Trends in Cognitive Sciences, 9(9), 445-453.

Wheeler, M. E., Petersen, S. E., \& Buckner, R. L. (2000a). Memory's echo: Vivid remembering reactivates sensory-specific cortex. Proceedings of the National Academy of Sciences of the United States of America, 20,11125-11129.

Wheeler, M. E., Petersen, S. E., \& Buckner, R. L. (2000b). Memory's echo: Vivid remembering reactivates sensory-specific cortex. Proceedings of the National Academy of Sciences of the United States of America, 97(20), 11125-11129.

Yonelinas, A. P., Otten, L. J., Shaw, K. N., \& Rugg, M. D. (2005). Separating the brain regions involved in recollection and familiarity in recognition memory. Journal of Neuroscience, 25(11), 3002-3008. 